\title{
STATINS-do we know them or are we Alice in wonderland?
}

\begin{abstract}
Statins are a major cornerstone in preventing cardiovascular diseases. Evidence from Clinical trials have clearly indicated that statins are beneficial in the primary and secondary prevention of coronary heart disease. However, the overall benefits observed with statins appear to be greater than what might be expected from changes in lipid levels alone, suggesting effects beyond cholesterol lowering. Recent studies have clearly indicated that some of the cholesterol-independent or "pleiotropic" effects of statins involve improving endothelial function, enhancing the stability of atherosclerotic plaques, decreasing oxidative stress and inflammation, and inhibiting the thrombogenic response. Pleiotropic effects of a drug are actions other than those for which the agent was specifically developed. These effects may be related or unrelated to the primary mechanism of action of the drug, and they are usually unanticipated. The results of several ongoing clinical trials aimed more specifically at pleiotropic effects should enlighten us on their relative clinical relevance and importance.
\end{abstract}

Keywords: statins, cardiovascular disease, dyslipidemia, atheroscelerosis, cholesterol
Volume 5 Issue 3 - 2017

\author{
Mrinal Ranjan Srivastava,' Anu Chandra² \\ 'Department of Community Medicine, India \\ ${ }^{2}$ Department of Biochemistry Era, India
}

Correspondence: Mrinal Ranjan Srivastava, Diploma family Medicine, Fellowship In Diabetes, Royal Liverpool academy, Std, India, Email dr.mrinal.srivastava@gmail.com

Received: May 27, 2017 | Published: September 6, 2017
Abbreviations: CVD, cardiovascular disease; FDA, food and drug administration; T2DM, type 2

diabetes; LDL-C, low density lipoprotein cholesterol; CKD, Chronic Kidney Disease; NAFLD, non-alcoholic fatty liver disease; OSAS, obstructive sleep apnea syndrome; COPD, chronic obstructive pulmonary disease; SLE, systemic lupus erythematosus.

\section{Introduction}

One of the most important discoveries of our times statins are a breakthrough in the treatment of high serum cholesterol. Infact different trials have indicated that statins substantially reduces both morbidity and mortality from coronary heart disease. Statins are now recognized as the mainstay in the management of patients with established Coronary Heart Diseases and these also hold promise for patients with high risk without evident Coronary Heart Diseases.

Cardiovascular disease (CVD) is the leading cause of worldwide deaths, with mortality rates of approximately 235 per 100,000 inhabitants. ${ }^{1}$ A vast majority of the cardiovascular diseases is attributed to Atheroscelerosis. Lovastatin became the first commercially available statin medication in 1987 when it was given the United States Food and Drug Administration (FDA) approval. ${ }^{2}$ Statins are the cornerstone of management of dyslipidemias, however there are still several controversies. Type 2 diabetes (T2DM) and statins link was first indicated in the Finnish study of 9000 men and it was indicated that people treated with statins had $46 \%$ more likely to develop diabetes however this was debatable as the researchers from Finland had used only men and white people how would this apply to women and people of other ethnic groups are questionable. Accordingly, statin treatment-associated T2DM should not affect management decisions. An increase in the use of statins is also thought to arise from their cholesterol-independent (pleiotropic) effects, which have implications in a wide variety of disease processes and thus may significantly broaden their therapeutic use. ${ }^{3}$

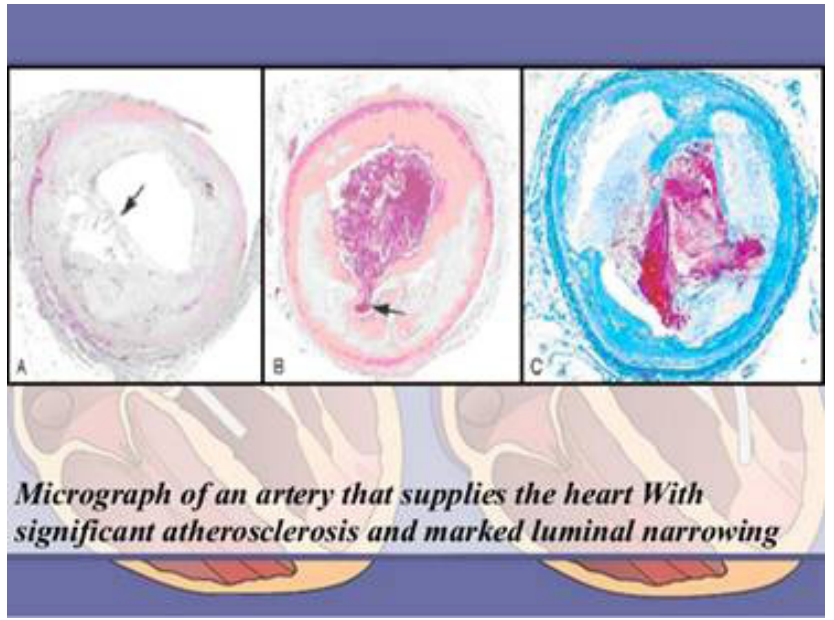

\section{Statin resistance and statin intolerance}

Many patients treated with statins are considered statin-resistant because they fail to achieve adequate reduction of low density lipoprotein cholesterol (LDL-C) levels. Some patients are statinintolerant because they are unable to tolerate statin therapy at all or to tolerate a full therapeutic statin dose because of adverse effects, particularly myopathy and increased activity of liver enzymes.

While statins have proven to be advantageous for lowering the LDL-C levels in the majority of individuals, some individuals fail to respond to treatment (statin resistant) or are prone to developing AEs (statin intolerant). ${ }^{4,5}$ Patients response to satins varies from 5\% to $70 \%$.Statin resistant patients do not respond to statin therapies. Statin resistance likely arises from a number of mechanisms including polymorphisms in genes involved in cholesterol synthesis and metabolism, such as 3-hydroxy-3-methyl-glutaryl-coenzyme A reductase (HMGCR) and low-density lipoprotein receptor (LDL-R), and those associated with statin pharmacokinetics, such as transporter 
proteins (eg, ATP-binding cassette sub-family G member 2 ABCG2 and SCLO1B1). Patients who are statin intolerant typically exhibit sensitivity to statin-induced myopathy and/or liver injury as indicated by increases in liver enzyme activity. In both these groups of patients other avenues for lowering of LDL-C must be sought after. Alternatives to statin therapy include use of cholesterol absorption inhibitors and bile-acid sequestrants; however, they lack the potency of statins in their ability to lower LDL-C levels. PCSk9 inhibitors are a newer treatment modality in those of high risk patient groups. ${ }^{6}$ If a patient is really statin-resistant or statin-intolerant, several other treatment possibilities are nowadays available: ezetimibe alone or in combination with bile acid sequestrants, and possibly in the near future mipomersen, lomitapide, or monoclonal antibodies against PCSK9 can be the newer treatment modalities.

\section{Adverse effects of use of statins}

While statins, in general, are well tolerated, AEs are reported and include muscle pain and damage, increased blood glucose levels, which may contribute to type 2 diabetes mellitus (T2DM), hepatotoxicity, digestive problems, cognitive effects, and the development of rashes or flushing. 24 Of these AEs, those impacting muscle, blood glucose levels, and liver function are thought to be the most clinically relevant.

\section{Type 2 diabetes}

The American Diabetes Association states that the benefits of taking a statin outweigh the risk of acquiring diabetes. The FDA still believes in the benefits of statins. The 2012 announcement isn't meant to urge all people to stop taking statins. Rather, it advises doctors to monitor the blood sugar levels of their patients who take these drugs. The diabetogenic effects of statins are thought to arise from several mechanisms that converge on glucose regulation and pancreatic beta cells. ${ }^{2-9}$ Including inhibition ofisoprenoid synthesisand subsequent inhibition of glucose uptake by beta cells, increased uptake of LDLleading to glucokinase inhibition(hence blocking glucose conversion to pyruvate), and cytokine-induced overproduction of nitric oxide (NO) leading to beta cell apoptosis. In addition, statins suppress ubiquinone and ATP synthesis. The ultimate effect of all these is the suppression of insulin production. Large no of studies have eventually reported that as compared to the placebo group a greater no of patients eventually receiving statins therapy were subsequently diagnosed with Diabetes. Statins may have a Diabetogenic potential. While new-onset T2DM is observed with all statins and associations coupled from those observations, a causal relationship cannot be implicated as numerous patients of the control group (receiving a placebo) also in fact developed T2DM. So summing up Statins therapy may be associated with slight increased risk of Type 2 Diabetes however if we compare it to the reduction in adverse coronary events statins therapy benefits outweighs the risk. Apart from acting on diabetic dyslipidemia, statins were shown to exert beneficial effects on several diabetic complications as well as other cardiovascular (CVD) risk predictors such as endothelial dysfunction, inflammation, oxidative stress, chronic kidney disease (CKD), non-alcoholic fatty liver disease (NAFLD), metabolic syndrome (MetS), obstructive sleep apnea syndrome (OSAS) and hyperuricemia.

\section{Liver function}

Statin induced Liver injury is usually mild to moderate and is reversible. It usually occurs after long term use usually months to years. Statin induced Hepatotoxicity is usually a rare phenomenon. The increased Transaminases levels are usually temporary. The increased transaminases levels usually occur due to increased membrane permeability and leakage of Liver enzymes. According to the FDA the currently marketed statins have a very Low risk of liver injury. Large trials of statins have shown no difference in the frequency or degree of ALT increases between treatment and placebo groups. Outof-range values, which do occur with statin use, eventually return to normal even if the same statin is continued. The occurrence of acute liver failure thought to be caused by statins is well below what is now understood as the background rate of idiopathic acute liver failure in the general population. No consistent liver- biopsy picture from possible statin-related drug injury has emerged, and there are no reports of chronic carriers of drug-induced liver damage from statins. Thus, an increased ALT in this situation is not a disease.

\section{Statins and skeletal muscle function}

Muscle associated symptoms in statins treated individuals occurs in $25 \%-30 \%$ of individuals. Patients may present with symptoms of soreness, aching and even stiffness. Statin-induced myopathy may be multifactorial, the result of impaired signal transduction, cell trafficking, gene transcription, structural protein formation and regulation, and oxidative phosphorylation. Clinically, these symptoms can be defined as myopathy (muscle weakness), myositis (muscle inflammation), myonecrosis (elevated muscle enzyme levels), rhabdomyolysis (severe myonecrosis with myoglobinuria or acute renal failure) and myalgia (unexplained muscle discomfort which encompasses muscle aches, soreness, etc). Commonly prescribed statins are identified by their lipophilic or hydrophilic nature Mild myalgia occurs in $5 \%-10 \%$ of statin users annually and is often intermittent.

\section{Pleiotropic effects of statins}

Statins have been found to have pleiotropic effects. The pleiotropic effects associated with statins have been found to have an impact on disease pathophysiology include their modulation of immune responses, their enhancement of anti-inflammatory processes, and their alterations of signaling pathways. The multitude of diseases linked to the pleiotropic effects of statins include multiple sclerosis (MS), inflammatory bowel diseases (IBDs), rheumatoid arthritis (RA), systemic lupus erythematosus (SLE), chronic obstructive pulmonary disease (COPD), cancer, strokes, Parkinson's and Alzheimer's diseases, bacterial infections, and HIV. The pleiotropic effects of statins were first reported in 1995 when it was observed that the benefits of pravastatin extended beyond those attributed to the expected lipid-lowering effects of statin therapy ${ }^{10,11}$. Statins have been found to stabilize atherosclerotic plaque, enhance endothelial function, decreases oxidative stress and modulate endothelial responses. Pleiotropic effects of statins include improvement of endothelial dysfunction, increased nitric oxide bioavailability, antioxidant properties, inhibition of inflammatory responses, and stabilization of atherosclerotic plaques. These and several other emergent properties could act in concert with the potent low-density lipoprotein cholesterol-lowering effects of statins to exert early as well as lasting cardiovascular protective effects. Understanding the pleiotropic effects of statins is important to optimize their use in treatment.

\section{Statins and chronic inflammatory diseases}

Rheumatoid arthritis-RA is characterized by chronic inflammation of the joints, synovial hyperplasia, bone destruction, joint deformity, and systemic inflammation. ${ }^{12}$ Patients with RA are at 
an increased risk of mortality, as much as $50 \%$, due primarily to increases in CVDs. A recent meta-analysis involving 992 RA patients demonstrated that statin use significantly decreased serum levels of inflammatory markers. ${ }^{13}$ Furthermore, patients taking statins have a reduced incidence of RA, and in those taking simvastatin and atorvastatin, a reduction in markers indicative of both RA and CVD has been reported. High-intensity statin treatment was associated with a $23 \%$ reduced risk of RA when compared with low-intensity statin treatment. Statins have been shown to reduce the level of CRP in patients with RA, which indicates anti-inflammatory effect of these agents, independent of their cholesterol lowering effects.

Statins and Inflammatory bowel diseases-A know of animal studies have demonstrated a decrease in inflammatory markers of the diseases with statin therapy. Statins use was also associated with the decrease in the dose of corticosteroids used in the treatment of inflammatory bowel diseases. Studies have also indicated that IBD patients on daily atorvastatin have significantly lower levels of C-Reactive proteins a marker for chronic inflammation.

Statins and COPD-The pathology of COPD, an irreversible and progressive obstruction of airflow, also involve an abnormal inflammatory response. While statins have been considered for use in treating COPD, current guidelines do not recommend the use of statins to prevent acute disease exacerbations.

\section{Statins and cancer}

Reduction in the incidence of Colorectal cancers with Statin use was observed and this led to the speculation that statins reduces the incidence of certain cancers. There have also been reports of Statins preventing the onset of cancer and inhibiting cancer metastasis and improving patient survival. With respect to breast cancer some studies have found no association between statin use and Breast cancer mortality whereas some studies have found benefit from statin use. There have been more positive reports on prostate cancer and some reports suggest statins prevents prostate cancer progression and mortality.

\section{Conclusion}

Before considering Statins as the jack of all trades we need more substantial evidence. However the pleiotropic role of statins have opened new realm of possibilities. The beneficial effects of statins are assumed to result from their ability to reduce cholesterol biosynthesis. However, because mevalonic acid is the precursor not only of cholesterol, but also of many nonsteroidal isoprenoid compounds, inhibition of 3-hydroxy-3-methylglutaryl coenzyme A reductase may result in pleiotropic effects. It has been shown that several statins decrease smooth muscle cell migration and proliferation there are also some caveats in the use of statins. We need to identify the cohort of population who would definitely benefit from the effects of statins. The effective type, dose and duration of therapy need to be identified. We need biomarkers for the pleiotropic effects of statins and the measured response of the statins. There is certainly a lack of evidence on the purported benefits of statin use. We need randomized controlled trials for additional evidence. Till then there would always be certain grey areas in the use of statins. The pleiotropic effects of statins and other drugs are under continued investigation to fully establish their role in the prevention of cardiovascular events. The results of several ongoing clinical trials aimed more specifically at pleiotropic effects should enlighten us on their relative clinical relevance and importance.

\section{Acknowledgments}

None.

\section{Conflicts of interest}

The authors declare that there are conflicts of interest.

\section{Funding}

None.

\section{References}

1. Barquera S, Pedroza-Tobías A, Medina C, et al. Global overview of the epidemiology of atherosclerotic cardiovascular disease. Arch Med Resm. 2015;46(5):328-338.

2. Endo A. A historical perspective on the discovery of statins. Proc Jpn Acad Ser B Phys Biol Sci. 2010;86(5):484-493.

3. Liao JK, Laufs U. Pleiotropic effects of statins. Annu Rev Pharmacol Toxicol. 2005;45:89-118

4. Wilson PW, Abbott RD, Castelli WP. High density lipoprotein cholesterol and mortality. The Framingham Heart Study. Arteriosclerosis. 1988;8(6):737-741.

5. Hobbs FD. Cardiovascular disease: different strategies for primary and secondary prevention? Heart. 2004;90(10): 1217-1223.

6. Dadu RT, Ballantyne CM. Lipid lowering with PCSK9 inhibitors. Nat Rev Cardiol. 2014;11(10):563-575.

7. Golomb BA, Evans MA. Statin adverse effects: a review of the literature and evidence for a mitochondrial mechanism. Am J Cardiovasc Drugs. 2008;8(6):373-418.

8. Sampson UK, Linton MF, Fazio S. Are statins diabetogenic? Curr Opin Cardiol. 2011;26(4):342-347.

9. Banach M, Malodobra-Mazur M, Gluba A, et al. Statin therapy and new-onset diabetes: molecular mechanisms and clinical relevance. Curr Pharm Des. 2013;19(27):4904-4912.

10. Shepherd J, Cobbe SM, Ford I, et al. Prevention of coronary heart disease with pravastatin in men with hypercholesterolemia. West of Scotland Coronary Prevention Study Group. $N$ Engl J Med. 1995;333(20):1301-1307.

11. Sacks FM, Pfeffer MA, Moye LA, et al. The effect of pravastatin on coronary events after myocardial infarction in patients with average cholesterol levels. Cholesterol and Recurrent Events Trial investigators. N Engl J Med. 1996;335(14):1001-1009.

12. Picerno V, Ferro F, Adinolfi A, et al. One year in review: the pathogenesis of rheumatoid arthritis. Clin Exp Rheumatol. 2015;33(4):551-558.

13. Lv S, Liu Y, Zou Z, et al. The impact of statins therapy on disease activity and inflammatory factor in patients with rheumatoid arthritis: a metaanalysis. Clin Exp Rheumatol. 2015;33(1):69-76. 